\title{
MORE ON CONVEX FUNCTIONS OF BOUNDED TYPE
}

\author{
A. W. GOODMAN
}

\begin{abstract}
A normalized univalent function is convex of bounded type if the curvature of the curve bounding the image domain lies between two fixed positive numbers. In this work we find sharp bounds for the modulus of the function and its derivative. We also discuss the coefficient problem for this new class of functions.
\end{abstract}

1. Introduction. In [2] the author introduced a new set of univalent functions denoted by $C V\left(R_{1}, R_{2}\right)$. Roughly speaking, these are the normalized functions

$$
f(z)=z+a_{2} z^{2}+a_{3} z^{3}+\cdots
$$

which are regular, univalent, and convex in the unit disk $E:|z|<1$, and for which $f(E)$ is a convex domain with radius of curvature $\rho \in\left[R_{1}, R_{2}\right]$ on $\partial F(E)$. In $[2]$ we found the Koebe domain, the sharp bounds for $d=\min \left|f\left(e^{i \theta}\right)\right|$, and we mentioned a few open problems for the set $C V\left(R_{1}, R_{2}\right)$. In this paper we obtain a few more theorems about this new set.

2. Bounds for $\left|f^{\prime}(z)\right|$ and $|f(z)|$. We begin with

THEOREM 1. If $f(z) \in C V\left(R_{1}, R_{2}\right)$ and $z=r e^{i \theta}$, then

$$
\left|f^{\prime}(z)\right| \leq R_{2} /\left(1-r^{2}\right) .
$$

This bound is sharp for each $r$ in $(0,1)$.

PROOF. By a rotation $e^{-i \alpha} f\left(z e^{i \alpha}\right)$ we may assume that $z=r>0$. Along with $f(z)$ we consider the function

$$
\phi(z) \equiv f\left(\frac{z+r}{1+r z}\right)
$$

for fixed $r$ in $(0,1)$. Now $\phi(E)=f(E)$ and from $[2,3]$ this domain is contained in a disk of radius $R_{2}$. If $\phi(z)=b_{0}+b_{1} z+b_{2} z^{2}+\cdots$, then the area theorem gives

$$
\sum_{n=0}^{\infty} n\left|b_{n}\right|^{2} \leq R_{2}^{2}
$$

and in particular $\left|b_{1}\right| \leq R_{2}$. Since

$$
\phi^{\prime}(0)=f^{\prime}(r)\left(1-r^{2}\right)=b_{1},
$$

this gives the inequality (2.1). This inequality is sharp for each $z$ in $E$, but the extremal function depends on $|z|=r$. Set

$$
r=\sqrt{1-1 / R_{2}}, \quad R_{2}=1 /\left(1-r^{2}\right),
$$

Received by the editors December 26, 1984 and, in revised form, June 24, 1985. Presented to the Society, January 1985 in Anaheim, California.

1980 Mathematics Subject Classification. Primary 30C45; Secondary 30C50.

Key words and phrases. Univalent, convex, bounded type, coefficient problem. 
so that for each $r$ in $(0,1)$ there is a unique $R_{2}$ in $(1, \infty)$ and conversely. Consider the extremal function $F(z)=z /(1-A z)$ for fixed $A$ in $(0,1)$. Now $F(E)$ is a disk of radius $1 /\left(1-A^{2}\right)$ centered at $A /\left(1-A^{2}\right)$ and hence $F(z)$ is in $C V\left(R_{1}, R_{2}\right)$ for every $R_{1} \leq R_{2}$ if $R_{2}=1 /\left(1-A^{2}\right)$. For fixed $r>0$ we set $A=r$ and find that

$$
F^{\prime}(r)\left(1-r^{2}\right)=\frac{1}{(1-A r)^{2}}\left(1-r^{2}\right)=\frac{1-r^{2}}{\left(1-r^{2}\right)^{2}}=\frac{1}{1-r^{2}}=R_{2} \text {. }
$$

We observe that the extremal function depends on the point $z$ of interest. Hence the bound (2.1) appears to be useless for finding a sharp bound for $|f(z)|$.

If we follow the technique used in proving Theorem 1 , but replace $\left|b_{1}\right| \leq R_{2}$ with $\left|b_{2}\right|=\left|\phi^{\prime \prime}(0)\right| / 2 \leq R_{2} / \sqrt{2}$ from (2.2) we will arrive at

$$
\left|\left(1-|z|^{2}\right)^{2} f^{\prime \prime}(z)-2 \bar{z}\left(1-|z|^{2}\right) f^{\prime}(z)\right| \leq \sqrt{2} R_{2} \text {. }
$$

However, this inequality does not appear to be sharp.

THEOREM 2. Let $d=\min \left|f\left(e^{i \theta}\right)\right|, 0 \leq \theta \leq 2 \pi$. If $f(z)$ is in $C V\left(R_{1}, R_{2}\right)$, then

$$
\left|f\left(r e^{i \theta}\right)\right| \leq \frac{r d\left(2 R_{2}-d\right)}{R_{2}(1-r)+r d} .
$$

This inequality is sharp for every $R_{2}$ in $(1, \infty)$ if $d=R_{2}-\sqrt{R_{2}^{2}-R_{2}}$.

PROOF. As we observed in [2] we can always rotate the image domain so that $f(E)$ is covered by a disk with diameter end points at $-d$ and $2 R_{2}-d$. Then we have the subordination

$$
f(z) \prec B z /(1-A z)
$$

where

$$
A=\left(R_{2}-d\right) / R_{2} \quad \text { and } \quad B=\left(2 R_{2}-d\right) d / R_{2} .
$$

Since the inverse of $w=B z /(1-A z)$ is $w=z /(A z+B)$, the function $\phi(z) \equiv$ $f(z) /(A f(z)+B)$ satisfies the conditions of the Schwarz lemma. Hence $|f(z)| \leq$ $r(A|f(z)|+B)$ for $|z|=r$. This gives $|f(z)| \leq r B /(1-r A)$. When (2.6) is used in this inequality, we arrive at (2.4).

To prove that (2.4) is sharp we use the same example function $F(z)=z /(1-A z)$ that was used in Theorem 1 . For this function $d=1 /(1+A)$ and $R_{2}=1 /\left(1-A^{2}\right)$. With these values a brief computation gives

$$
\frac{r d\left(2 R_{2}-d\right)}{R_{2}(1-r)+r d}=\frac{r}{1-A r}=F(r)
$$

It would be nice to have a sharp bound on the right side of $(2.4)$ that is free of $d$, a bound that depends only on $R_{2}$ and $r$. To obtain such a bound we observe that the right side of $(2.4)$ has the form $r Q\left(r, R_{2}, d\right)$. Here $Q$ is a decreasing function of $d$ if $r^{*}<r<1$ where

$$
r^{*}=\frac{2 R_{2}\left(R_{2}-d\right)}{2 R_{2}\left(R_{2}-d\right)+d^{2}}<1 .
$$

From [2] we know that $d \geq R_{2}-\sqrt{R_{2}^{2}-R_{2}}$. Hence if $r^{*} \leq r<1$, we may replace 
$d$ by $R_{2}-\sqrt{R_{2}^{2}-R_{2}}$ in (2.4). This gives

THEOREM 3. If $f(z)$ is in $C V\left(R_{1}, R_{2}\right)$ and $|z|=r$ is in $\left[r^{*}, 1\right)$, then

$$
|f(z)| \leq r \frac{R_{2}}{R_{2}-r \sqrt{R_{2}^{2}-R_{2}}} .
$$

$A$ brief computation shows that this upper bound is sharp for $f(z)=z /(1-A z)$ when $A^{2}=1-1 / R_{2}$.

3. The coefficient problem. We know that among all functions in $C V\left(R_{1}, R_{2}\right)$, the area of $f(E)$ is greatest when $f(E)$ is a disk of radius $R_{2}$. This occurs for

$$
F(z)=\frac{z}{1-A z}=z+A z^{2}+A^{2} z^{3}+\cdots,
$$

when $A^{2}=1-1 / R_{2}$. Thus one might expect that in the class $C V\left(R_{1}, R_{2}\right)$ we should have the inequality

$$
\left|a_{k}\right| \leq A^{k-1}=\left(1-\frac{1}{R_{2}}\right)^{(k-1) / 2}, \quad k=2,3, \ldots,
$$

where $f(z)$ has the form (1.1). However, as we indicated in [2], the bound (3.2) cannot be correct for all $R_{2}>1$ and all $k \geq 2$. Here we look into this disappointment a little more closely. At present the true sharp bound for $\left|a_{k}\right|$ is not known, not even for $k=2$.

Among the competing functions to maximize $\left|a_{k}\right|$, it is natural to consider a function with only two nonzero coefficients, namely

$$
G(z) \equiv z+c_{k} z^{k} \equiv z+c z^{k}, \quad c>0, k \geq 2,
$$

where for simplicity we drop the subscript on $c$. Similarly for the same fixed $k$ we set $b=A^{k-1}>0$ in

$$
F(z)=\frac{z}{1-A z}=z+\cdots+b z^{k}+\cdots .
$$

Our object is to compare $b$ and $c$ when both $F(z)$ and $G(z)$ have the same max $\rho=$ $R_{2}$. For $F(z), R_{2}=\left(1-b^{2 /(k-1)}\right)^{-1}$, and for $G(z), R_{2}=(1-k c)^{2} /\left(1-k^{2} c\right)$ where $0 \leq k^{2} c<1$. Hence $b$ and $c$ are related by

$$
R_{2}=\frac{1}{1-b^{2 /(k-1)}}=\frac{(1-k c)^{2}}{1-k^{2} c}, \quad 0 \leq k^{2} c<1 .
$$

Simple manipulations give the equivalent equation

$$
b^{2 /(k-1)}=c \frac{k(k-2+k c)}{(1-k c)^{2}} .
$$

When $k=2$, equation (3.6) gives $b^{2}=4 c^{2} /(1-2 c)^{2}$, or $b=2 c /(1-2 c)$. Thus for all $R_{2}>1$, we have $b>2 c$.

When $k=3$, equation (3.6) gives

$$
b=c \frac{3(1+3 c)}{(1-3 c)^{2}}>3 c
$$


for all $R_{2}>1$. Thus we have

LEMMA 1. Let $G(z)=z+c z^{k}, 0<k^{2} c<1$, and let $F(z)=z /(1-A z)=$ $z+\cdots+b z^{k}+\cdots, A>0$. Suppose that both functions have the same $R_{2}>1$. If $k=2$ or $k=3$, then $b>c$.

It is worth noting that this simple inequality fails for $k \geq 4$. To see this we put (3.6) in the form

$$
b=c\left[c^{(k-3) / 2}\left(\frac{k(k-2+k c)}{(1-k c)^{2}}\right)^{(k-1) / 2}\right] \equiv c J(k, c) .
$$

Now $J(k, c)$ is obviously an increasing function of $c$ for $0 \leq c<1 / k^{2}$, and $k \geq 4$. Further $J(k, 0)=0$ and $J(k, c) \rightarrow k^{2} \geq 16$, as $c \rightarrow 1 / k^{2}$. Hence there is a unique $c^{*}$ in $\left(0,1 / k^{2}\right)$ such that $b=c$ in (3.7) and (3.6). For $0<c<c^{*}$, we have $b<c$, and for $c^{*}<c<1 / k^{2}$ we have $b>c$. Equation (3.5) determines a unique $R^{*}>1$ with the properties expressed in

LEMMA 2. Let $k \geq 4$. With the notation of Lemma 1, there is an $R^{*}$ with the following property. If $1<R_{2}<R^{*}$, then $b<c$. If $R_{2}>R^{*}$, then $c>b$.

These two lemmas indicate the difficulty of the coefficient problem in the set $C V\left(R_{1}, R_{2}\right)$ and may be of some slight help in the solution of this problem.

\section{REFERENCES}

1. A. W. Goodman, Univalent functions, Vols. I and II, Mariner Publishing Co., Tampa, Florida, 1983. (Address Book Publishers Inc., P.O. Box 21492, Tampa FL 33622)

2. _ C Convex functions of bounded type, Proc. Amer. Math. Soc. 92 (1984), 541-546.

3. - Convex curves of bounded type, Internat. J. Math. Math. Sci. 8 (1985), 625-636. 33620

Department of MAThematics, UNIVERSity of SOUTh Florida, TAMPA, FloRidA 